Chinese Journal of Organic Chemistry

ARTICLE

\title{
含取代苯甲酰基硫腿的核苷类化合物的合成及杀菌活性研究
}

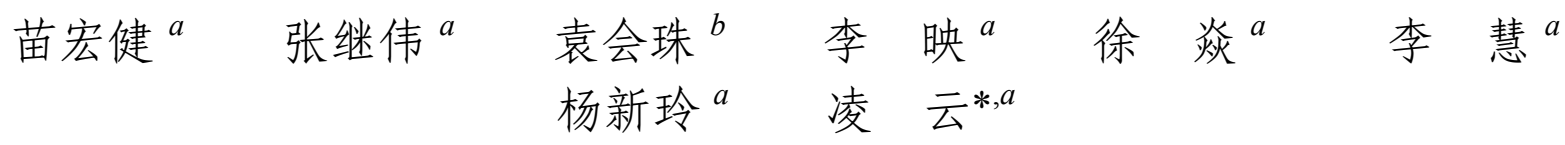

$\left({ }^{a}\right.$ 中国农业大学理学院应用化学系 农业部农药化学及应用重点开放实验室 北京 100193)

$\left({ }^{b}\right.$ 中国农业科学院植物保护研究所 北京 100193)

\begin{abstract}
摘要 为了发现具有杀菌活性的新型先导化合物, 基于几丁质合成酶催化作用机制, 通过活性亚结构拼接方法, 保留 多氧需素和尼克霉素中的活性尿苷部分, 将具有良好杀菌活性的硫腿基团引入, 设计合成了一系列含硫腿结构的核苷 类化合物. 以尿苷为原料, 经 5 步反应制得目标物，其结构经 IR, ${ }^{1} \mathrm{H}$ NMR 及元素分析确证. 初步生测结果表明, 部分化 合物对芦笋茎枯病 (Phomopsisasparagi bubak) 表现出明显的抑制活性，其中 $6 \mathrm{~m}$ 的抑制率在 $50 \mu \mathrm{g} / \mathrm{mL}$ 浓度下为 $97.2 \%$, 与相同浓度的多氧霉素 B 活性(100\%)接近.
\end{abstract}

关键词 几丁质; 多氧需素; 尿苷; 硫豚; 杀菌活性

\section{Synthesis and Fungicidal Activities of Nucleoside Compounds Containing Substituted Benzoyl Thiourea}

\author{
Miao, Hongjian ${ }^{a}$ \\ Zhang, Jiwei ${ }^{a}$ \\ Yuan, Huizhu ${ }^{b}$ \\ Li, Ying ${ }^{a}$ \\ $\mathrm{Xu}, \mathrm{Yan}^{a}$ \\ $\mathrm{Li}, \mathrm{Hui}^{a}$ \\ Yang, Xinling ${ }^{a} \quad$ Ling, Yun ${ }^{*, a}$ \\ $\left({ }^{a}\right.$ Key Laboratory of Pesticide Chemistry and Application, Ministry of Agriculture, Department of Applied Chemistry, \\ College of Science, China Agricultural University, Beijing 100193) \\ $\left({ }^{b}\right.$ Institute of Plant Protection, Chinese Academy of Agricultural Sciences, Beijing 100193)
}

\begin{abstract}
To find new fungicidal lead compounds, based on the catalytic mechanism of chitin synthase, a series of novel nucleoside compounds containing thiourea were designed via the method of linking active sub-structures, in which the thiourea with high fungicidal activity was combined to the uridine part of polyoxins and nikkomycins. The target compounds were synthesized from uridine in 5 steps. Their structures were confirmed by IR, ${ }^{1} \mathrm{H}$ NMR spectra and elemental analysis. The preliminary bioassay results indicated that some compounds showed obvious inhibition effects against Phomopsisasparagi bubak, especially, and the fungicidal activity of $6 \mathrm{~m}(97.2 \%)$ at $50 \mu \mathrm{g} / \mathrm{mL}$ is similar to that $(100 \%)$ of polyoxin B.
\end{abstract}

Keywords chitin; polyoxins; uridine; thiourea; fungicidal activity

几丁质(Chitin)是真菌细胞壁和昆虫表皮的特有成 分，不存在于植物和哺乳动物中，因而是开发选择性医 药或农药的理想靶标 ${ }^{[1]}$. 如作用于昆虫表皮形成的苯甲 酰艮类昆虫生长调节剂, 因具有选择性高、对环境友好 的特点，而成为害虫综合防治的有力工具之一 ${ }^{[2]}$. 但是, 作用于真菌细胞壁几丁质形成的农用杀菌剂尚不多见， 多氧霉素(polyoxins) ${ }^{[3]}$ 和尼克霉素(nikkomycins) ${ }^{[4]}$ 两类 核苷肽类化合物是典型的抑制真菌细胞壁形成的几丁
质合成抑制剂. 但因其细胞穿透性差、易酶解等不足而 限制了它们的应用 ${ }^{[5]}$. 因此，针对真菌几丁质形成开发 结构新颖、稳定性高且安全的农用杀菌剂具有重要的意 义.

几丁质是由 $\beta$-1,4-糖苷键连接的 $N$-乙酰氨基葡萄糖 多聚体，由几丁质合成酶(chitin synthase, CS)催化底物 尿苷二磷酸- $N$-乙酰氨基葡萄糖(UDP-GlcNAc) 生成 ${ }^{[6]}$. 研究表明 ${ }^{[7]}, \mathrm{CS}$ 结构中存在两个活性作用位点,一个是

\footnotetext{
* E-mail: lyun@cau.edu.cn, Tel.: 86-10-62732223.

Received October 11, 2011; revised November 26, 2011; published online January 10, 2012.

Project supported by the National Natural Science Foundation of China (No. 21072222), the National Basic Research Program of China (973 Program) (No. 2010CB126104), and the National "Twelfth Five-Year" Plan for Science \& Technology Support (No. 2011BAE06B05-5).

国家自然科学基金(No. 21072222)、国家 973 计划(No. 2010CB126104)和“十二五”科技支撑计划(No. 2011BAE06B05-5)资助项目.
} 
核苷结合位点; 另外一个是氨基酸催化位点. 多氧霉素 结构中的核苷部分能与 CS 的核苷结合位点相结合, 与 CS 底物产生竞争性作用; 而与核苷相连的侧链可能模 拟底物的磷酸键与 $\mathrm{Mg}^{2+}, \mathrm{Mn}^{2+}$ 等结合, 从而竞争性地干 扰 CS 催化底物 UDP-GlcNAc. 所以, 多氧霉素被认为是 底物 UDP-GlcNAc 的竞争物而干扰几丁质的正常形成. $\mathrm{Obi}^{[8]}$, Sunita 等 ${ }^{[9]}$ 基于上述作用机制, 以多氧霉素或尼 克霉素为先导, 设计合成的化合物对多种病原菌都具有 较好的抑制活性.

为了发现结构新颖、作用机制独特的杀菌剂先导化 合物, 本文在课题组前期工作的基础上 ${ }^{[10]}$, 基于几丁质 合成酶的结合和催化作用特点, 保留底物 UDP-GlcNAc 和多氧霉素、尼克霉素结构中共有的尿苷部分, 并在尿 苷的 2',3'-羟基处引入亚异丙基增加其脂溶性，进而增 加药物对病原菌体表的穿透性, 同时将具有良好杀菌活 性且能与金属离子易结合的硫脲基团 ${ }^{[11]}$ 与尿苷连接, 设计合成了一系列含硫脲基团的核苷类化合物(目标物 的设计策略见 Scheme 1), 并对其进行了杀菌活性初篮.
目标物的合成路线如 Scheme 2 所示.

\section{1 实验部分}

\section{1 仪器和试剂}

Cole-Parmer 熔点仪(温度计未校正); Bruker Advance DPX $300 \mathrm{MHz}$ 核磁共振仪 (以 $\mathrm{TMS}$ 为内标, DMSO- $d_{6}$ 为溶剂); PerkinElmer Spectrum 100 FT-IR 红外 光谱仪 ( $\mathrm{KBr}$ 压片); Vario EL III 型元素分析仪(Flash EA 1112 分析方法). 2',3'-异丙亚甲基-5'-脱氧氨基尿芺(4)参 照文献[12]方法制备，取代苯甲酰基异硫氧酸酯(5)参照 文献[13]方法制备. 所用试剂均为市售分析纯或化学纯 试剂, 溶剂用前均经无水处理.

\section{2 目标物 $N$-苯甲酰基- $N^{\prime}-\left(2^{\prime}, 3^{\prime}-O\right.$-异亚丙基-5'-脱氧} 尿苷)硫脲(6)的合成

1.2.1 中间体 $2^{\prime}, 3^{\prime}$-异丙亚甲基-5'-脱氧氨基尿苷(4)的 制备

$500 \mathrm{~mL}$ 反应瓶中, 加入 $24.4 \mathrm{~g}(0.1 \mathrm{~mol})$ 尿苷、 $1.72 \mathrm{~g}$

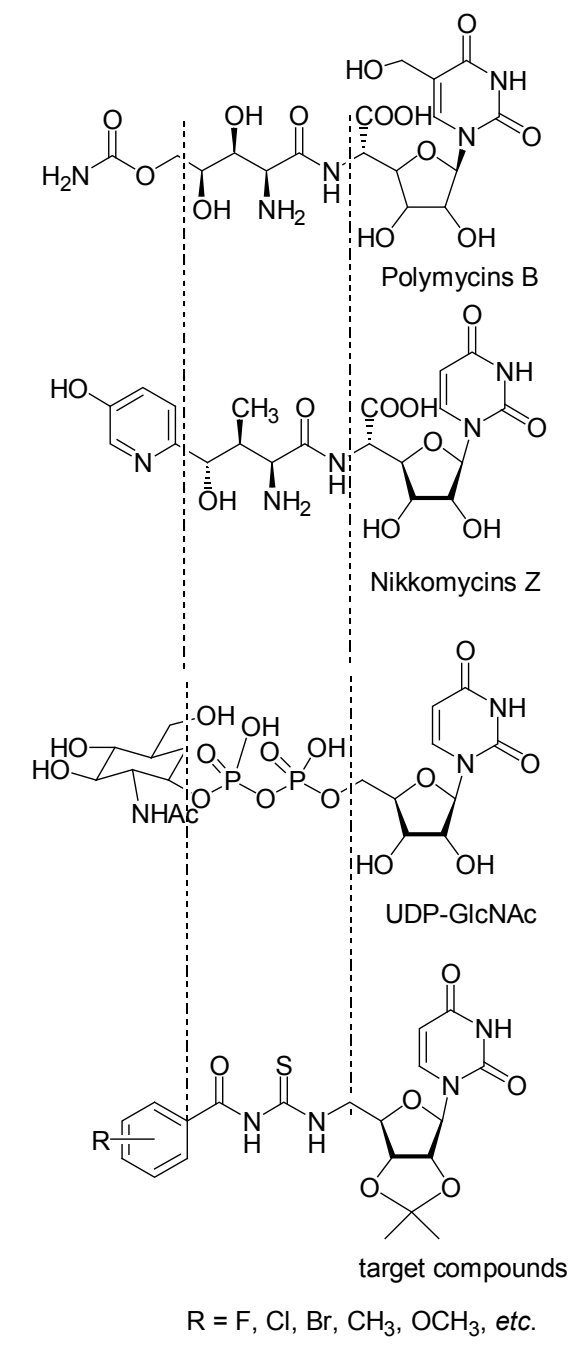

Scheme 1 


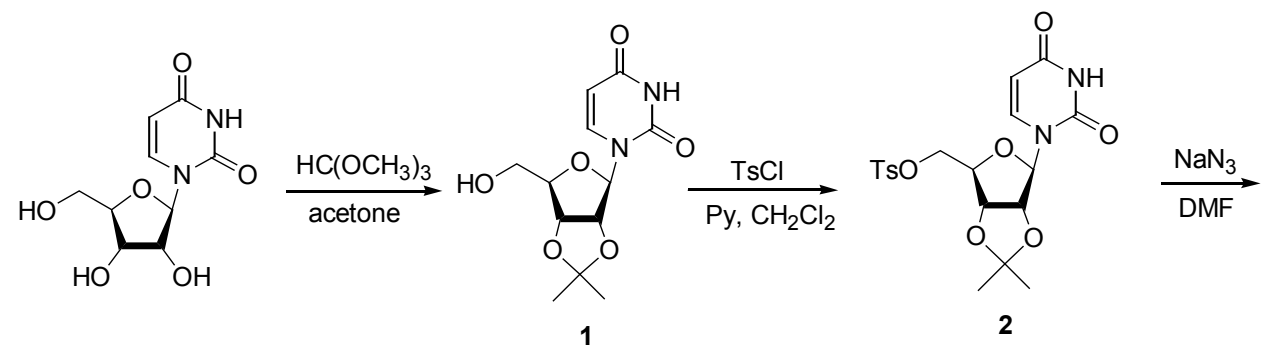<smiles></smiles>

Scheme 2

(0.01 mol)对甲苯磺酸和 $300 \mathrm{~mL}$ 丙酮, 滴加 $11.1 \mathrm{~g}(0.1$ 反应瓶内出现大量白色固体, 加入丙酮使固体全部溶 解, 加入 $8.40 \mathrm{~g}(0.1 \mathrm{~mol})$ 碳酸氢钠, 室温下摚拌 $30 \mathrm{~min}$, 将反应液中固体滤出. 滤液减压脱溶后得白色固体, 干 燥后得 $28.4 \mathrm{~g}$ 化合物 $2^{\prime}, 3^{\prime}-O$-异亚丙基尿苷(1), 收率 $99.8 \%$.

$500 \mathrm{~mL}$ 反应瓶中, 将 $28.4 \mathrm{~g}(0.1 \mathrm{~mol})$ 化合物 1 溶于 $80 \mathrm{~mL}$ 吡啶与 $250 \mathrm{~mL}$ 二氯甲烷混合溶液中, 加入 $19.1 \mathrm{~g}$ (0.1 mol) 对甲苯磺酰氯, 回流反应 $6.0 \mathrm{~h}$. 反应完毕后脱 溶得到淡黄色粘稠状液体, 将此液体倒入 $500 \mathrm{~mL}$ 冰水 中有淡黄色色固体析出, 抽滤, 固体烘干. 滤液用乙酸 乙酯 $50 \mathrm{~mL} \times 3$ 萃取, 合并有机相, 无水硫酸钠干燥, 脱 溶得淡黄色粘稠状液体, 此液体倒入 $200 \mathrm{~mL}$ 冰水中, 再次析出淡黄色固体, 抽滤后烘干. 将两次固 体合并得 $42.1 \mathrm{~g}$ 化合物 2',3'-O-异亚丙基-5'-对甲苯磺酰 基尿苷(2)，收率 $96.1 \%$.

$50 \mathrm{~mL}$ 反应瓶中加入 $12.7 \mathrm{~g}(0.03 \mathrm{~mol})$ 化合物 2,40 $\mathrm{mL} N, N$-二甲基甲酰胺和 $18.8 \mathrm{~g}(0.29 \mathrm{~mol})$ 叠氮化钠, 50 ${ }^{\circ} \mathrm{C}$ 反应 $8.0 \mathrm{~h}$. 然后将反应液倒入 $150 \mathrm{~mL}$ 冰水中, 充分摚 拌, 静置, 有乳白色固体析出, 干燥后得 $8.51 \mathrm{~g}$ 化合物 2',3'-O-异亚丙基-5'-叠氮基-5'-脱氧尿苷(3), 收率 95.1\%.

$100 \mathrm{~mL}$ 单口瓶中加入 $4.31 \mathrm{~g}(0.014 \mathrm{~mol})$ 化合物 3 $70 \mathrm{~mL}$ 甲醇和 $0.28 \mathrm{~g} 10 \%$ 的 $\mathrm{Pd} / \mathrm{C}$, 通入氢气, 室温下反 应 $2.0 \mathrm{~h}$. 然后滤去 $\mathrm{Pd} / \mathrm{C}$, 滤液减压脱溶后得粗产物, 甲 醇重结晶, 干燥得 $3.58 \mathrm{~g}$ 化合物 2',3'-O-异亚丙基-5'-氨 基-5'-脱氧尿苷(4), 收率 90.7\%.

\subsection{2中间体取代苯甲酰基异硫氧酸酯(5)的制备}

以苯甲酰基异硫氧酸酯为例. 在 $1000 \mathrm{~mL}$ 干燥三口 烧瓶中, 加入 $3.48 \mathrm{~g}(0.11 \mathrm{~mol})$ 硫氰酸钾和 $400 \mathrm{~mL}$ 无水 $\mathrm{mol}$ ) 原甲酸三甲酯, 室温下反应至反应液变澄清. 随后 乙腈, 搅拌溶解后缓慢滴加 $14.1 \mathrm{~g}(0.1 \mathrm{~mol})$ 苯甲酰氯的 $120 \mathrm{~mL}$ 无水乙腈溶液, 回流 $2 \mathrm{~h}$, 将固体滤出, 得苯甲 酰基异硫氰酸酯(收率为 76.5\%)的乙腈溶液备用.

\subsection{3 目标物 6 的合成}

以 $6 \mathbf{a}$ 为例, $250 \mathrm{~mL}$ 反应瓶中加入 $1.42 \mathrm{~g}(0.005 \mathrm{~mol})$ 化合物 4, 将其溶于 $100 \mathrm{~mL}$ 苯甲酰基异硫氰酸酯(5)的 乙腈溶液中, 加热回流 $2.0 \mathrm{~h}$. 将固体滤出, 滤液脱溶, 柱层析纯化, 展开剂: $V$ (石油醚) $: V$ ( 乙酸乙酯 $)=1$ :

1.3. 减压脱溶后得到 $1.21 \mathrm{~g} \mathrm{6a}$, 收率 $54.3 \%$.

用相同方法制得目标物 $\mathbf{6 b} \sim \mathbf{6 0}$, 所有化合物的理 化数据见表 1 、核磁数据见表 2 , 红外和元素分析数据见 表 3 .

\section{3 目标物 6 的杀菌活性测定}

对目标化合物采用菌丝生长速率法进行杀菌活性 测定 ${ }^{[14]}$. 供试菌种为棉花枯萎病 (Fusarium oxysporum)、 水稻纹枯病 (Rhizoctonia solani) 和芦笋茎枯病 (Phomopsisasparagi bubak). 分别称取 $50 \mathrm{mg}$ 目标化合 物, $157 \mathrm{mg} 34 \%$ 多氧霉素 B 原药, 然后将样品溶于二甲 亚砜, 分别定容成浓度为 $5000 \mu \mathrm{g} / \mathrm{mL}$ 的药液备用.

取 $1 \mathrm{~mL}$ 浓度为 $5000 \mu \mathrm{g} / \mathrm{mL}$ 的药液与 $99 \mathrm{~mL}$ 融化的 马铃薯葡萄糖琼脂(PDA)培养基混匀, 制备成浓度为 50 $\mu \mathrm{g} / \mathrm{mL}$ 含毒培养基 $100 \mathrm{~mL}$. 将含毒培养基平均分成 4 份, 分别倒入 4 个直径为 $9 \mathrm{~cm}$ 的培养血中, 制成含毒 PDA 平板. 待血中含毒培养基冷凝后, 分别接入培养好 的直径为 $0.5 \mathrm{~cm}$ 的病原菌菌饼. 置于 $25{ }^{\circ} \mathrm{C}$ 培养箱中培 养. 以相同浓度的多氧霉素 $\mathrm{B}$ 为对照药剂, 二甲亚砜为 溶剂对照, 同时设无菌水为空白对照, 每个样品 4 次重 复, 以上操作均为无菌操作. 待空白对照中的菌落充分 
表 1 目标化合物的理化数据

Table 1 Physical and chemical data of target compounds

\begin{tabular}{|c|c|c|c|c|}
\hline Compd. & $\mathrm{R}$ & Appearance & m.p. $/{ }^{\circ} \mathrm{C}$ & Yield/\% \\
\hline $6 a$ & $\mathrm{H}$ & 白色固体 & $146 \sim 147$ & 54.3 \\
\hline $6 b$ & $2-\mathrm{F}$ & 白色固体 & $194 \sim 195$ & 60.0 \\
\hline $6 c$ & $4-\mathrm{F}$ & 白色固体 & $191 \sim 192$ & 71.7 \\
\hline 6d & $2,6-\mathrm{F}_{2}$ & 白色固体 & $120 \sim 121$ & 67.1 \\
\hline $6 e$ & $3-\mathrm{Cl}$ & 白色固体 & $81 \sim 82$ & 24.2 \\
\hline $6 f$ & $4-\mathrm{Cl}$ & 白色固体 & $208 \sim 209$ & 70.9 \\
\hline $6 g$ & $2,4-\mathrm{Cl}_{2}$ & 白色固体 & $222 \sim 223$ & 56.4 \\
\hline $6 h$ & $2-\mathrm{Br}$ & 白色固体 & $191 \sim 192$ & 51.1 \\
\hline $6 \mathbf{i}$ & $2-\mathrm{NO}_{2}$ & 淡黄色固体 & $214 \sim 215$ & 65.7 \\
\hline $6 \mathbf{j}$ & $4-\mathrm{NO}_{2}$ & 白色固体 & $225 \sim 227$ & 65.1 \\
\hline $6 \mathbf{k}$ & $2-\mathrm{OCH}_{3}$ & 白色固体 & $110 \sim 111$ & 54.6 \\
\hline 61 & $4-\mathrm{OC}_{2} \mathrm{H}_{5}$ & 白色固体 & $184 \sim 185$ & 67.7 \\
\hline $6 \mathrm{~m}$ & $2-\mathrm{CH}_{3}$ & 白色固体 & $194 \sim 195$ & 65.7 \\
\hline $6 n$ & $4-\mathrm{C}\left(\mathrm{CH}_{3}\right)_{3}$ & 白色固体 & $152 \sim 153$ & 89.1 \\
\hline 60 & $4-\mathrm{Ph}$ & 白色固体 & $156 \sim 157$ & 43.8 \\
\hline
\end{tabular}

表 2 目标化合物的核磁数据

Table $2{ }^{1} \mathrm{H}$ NMR spectral data of target compounds

\begin{tabular}{|c|c|}
\hline Compd. & ${ }^{1} \mathrm{H}$ NMR (DMSO- $\left.d_{6}, 300 \mathrm{MHz}\right) \delta$ \\
\hline $6 a$ & $\begin{array}{l}11.46 \sim 11.44(\mathrm{~m}, 2 \mathrm{H}, \mathrm{NH}), 11.02(\mathrm{t}, J=5.31 \mathrm{~Hz}, 1 \mathrm{H}, \mathrm{NH}), 7.95 \sim 7.91(\mathrm{~m}, 2 \mathrm{H}, \mathrm{ArH}), 7.76(\mathrm{~d}, J=8.04 \mathrm{~Hz}, 1 \mathrm{H}, 6-\mathrm{H}), \\
7.67 \sim 7.61(\mathrm{~m}, 1 \mathrm{H}, \mathrm{ArH}), 7.54 \sim 7.48(\mathrm{~m}, 1 \mathrm{H}, \mathrm{ArH}), 5.83\left(\mathrm{~d}, J=2.07 \mathrm{~Hz}, 1 \mathrm{H}, 1^{\prime}-\mathrm{H}\right), 5.65(\mathrm{dd}, J=2.19,5.76 \mathrm{~Hz}, 1 \mathrm{H}, \\
5-\mathrm{H}), 5.09\left(\mathrm{dd}, J=2.13,4.35 \mathrm{~Hz}, 1 \mathrm{H}, 2^{\prime}-\mathrm{H}\right), 4.85\left(\mathrm{dd}, J=4.41,6.39 \mathrm{~Hz}, 1 \mathrm{H}, 3^{\prime}-\mathrm{H}\right), 4.34 \sim 4.28\left(\mathrm{~m}, 1 \mathrm{H}, 4^{\prime}-\mathrm{H}\right), 4.06 \sim \\
3.92\left(\mathrm{~m}, 2 \mathrm{H}, 5^{\prime}-\mathrm{H}\right), 1.49\left(\mathrm{~s}, 3 \mathrm{H}, \mathrm{CH}_{3}\right), 1.300\left(\mathrm{~s}, 3 \mathrm{H}, \mathrm{CH}_{3}\right)\end{array}$ \\
\hline $6 \mathbf{b}$ & $\begin{array}{l}11.49(\mathrm{~s}, 1 \mathrm{H}, \mathrm{NH}), 11.40(\mathrm{~d}, J=1.98 \mathrm{~Hz}, 1 \mathrm{H}, \mathrm{NH}), 10.75(\mathrm{t}, J=5.55 \mathrm{~Hz}, 1 \mathrm{H}, \mathrm{NH}), 7.81(\mathrm{~d}, J=8.04 \mathrm{~Hz}, 1 \mathrm{H}, 6-\mathrm{H}), \\
7.70 \sim 7.59(\mathrm{~m}, 2 \mathrm{H}, \mathrm{ArH}), 7.39 \sim 7.29(\mathrm{~m}, 2 \mathrm{H}, \mathrm{ArH}), 5.82\left(\mathrm{~d}, J=2.01 \mathrm{~Hz}, 1 \mathrm{H}, 1^{\prime}-\mathrm{H}\right), 5.64(\mathrm{dd}, J=2.13,7.95 \mathrm{~Hz}, 1 \mathrm{H}, \\
5-\mathrm{H}), 5.09\left(\mathrm{dd}, J=1.95,6.42 \mathrm{~Hz}, 1 \mathrm{H}, 2^{\prime}-\mathrm{H}\right), 4.85\left(\mathrm{dd}, J=4.41,6.42 \mathrm{~Hz}, 1 \mathrm{H}, 3^{\prime}-\mathrm{H}\right), 4.35 \sim 4.29\left(\mathrm{~m}, 1 \mathrm{H}, 4^{\prime}-\mathrm{H}\right), 4.04 \sim \\
3.89\left(\mathrm{~m}, 2 \mathrm{H}, 5^{\prime}-\mathrm{H}\right), 1.50\left(\mathrm{~s}, 3 \mathrm{H}, \mathrm{CH}_{3}\right), 1.30\left(\mathrm{~s}, 3 \mathrm{H}, \mathrm{CH}_{3}\right)\end{array}$ \\
\hline $6 c$ & $\begin{array}{l}11.54(\mathrm{~s}, 1 \mathrm{H}, \mathrm{NH}), 11.46(\mathrm{~s}, 1 \mathrm{H}, \mathrm{NH}), 10.95(\mathrm{t}, J=5.28 \mathrm{~Hz}, 1 \mathrm{H}, \mathrm{NH}), 7.95 \sim 7.92(\mathrm{~m}, 2 \mathrm{H}, \mathrm{ArH}), 7.75(\mathrm{~d}, J=8.07 \mathrm{~Hz}, \\
1 \mathrm{H}, 6-\mathrm{H}), 7.61 \sim 7.57(\mathrm{~m}, 2 \mathrm{H}, \mathrm{ArH}), 5.82\left(\mathrm{~d}, J=2.10 \mathrm{~Hz}, 1 \mathrm{H}, 1^{\prime}-\mathrm{H}\right), 5.65(\mathrm{~d}, J=7.98 \mathrm{~Hz}, 1 \mathrm{H}, 6-\mathrm{H}), 5.09(\mathrm{dd}, J=2.13, \\
\left.6.45 \mathrm{~Hz}, 1 \mathrm{H}, 2^{\prime}-\mathrm{H}\right), 4.85\left(\mathrm{dd}, J=4.41,6.42 \mathrm{~Hz}, 1 \mathrm{H}, 3^{\prime}-\mathrm{H}\right), 4.31\left(\mathrm{dd}, J=2.67,4.20 \mathrm{~Hz}, 1 \mathrm{H}, 4^{\prime}-\mathrm{H}\right), 4.00 \sim 3.93(\mathrm{~m}, 2 \mathrm{H}, \\
\left.5^{\prime}-\mathrm{H}\right), 1.50\left(\mathrm{~s}, 3 \mathrm{H}, \mathrm{CH}_{3}\right), 1.30\left(\mathrm{~s}, 3 \mathrm{H}, \mathrm{CH}_{3}\right)\end{array}$ \\
\hline $6 d$ & $\begin{array}{l}12.09(\mathrm{~s}, 1 \mathrm{H}, \mathrm{NH}), 11.44(\mathrm{~s}, 1 \mathrm{H}, \mathrm{NH}), 10.59(\mathrm{t}, J=5.28 \mathrm{~Hz}, 1 \mathrm{H}, \mathrm{NH}), 7.74(\mathrm{~d}, J=8.04 \mathrm{~Hz}, 1 \mathrm{H}, 6-\mathrm{H}), 7.64 \sim 7.58(\mathrm{~m}, \\
1 \mathrm{H}, \mathrm{ArH}), 7.22(\mathrm{t}, J=8.22 \mathrm{~Hz}, 2 \mathrm{H}, \mathrm{ArH}), 5.82\left(\mathrm{~d}, J=1.89 \mathrm{~Hz}, 1 \mathrm{H}, 1^{\prime}-\mathrm{H}\right), 5.63(\mathrm{dd}, J=1.53,7.92 \mathrm{~Hz}, 1 \mathrm{H}, 5-\mathrm{H}), 5.09 \\
\left(\mathrm{dd}, J=1.86,6.39 \mathrm{~Hz}, 1 \mathrm{H}, 2^{\prime}-\mathrm{H}\right), 4.86\left(\mathrm{dd}, J=4.29,6.24 \mathrm{~Hz}, 1 \mathrm{H}, 3^{\prime}-\mathrm{H}\right), 4.34\left(\mathrm{t}, J=3.57 \mathrm{~Hz}, 1 \mathrm{H}, 4^{\prime}-\mathrm{H}\right), 4.05(\mathrm{~d}, J= \\
\left.5.25 \mathrm{~Hz}, 1 \mathrm{H}, 5^{\prime}-\mathrm{H}\right), 3.91\left(\mathrm{t}, J=2.07 \mathrm{~Hz}, 1 \mathrm{H}, 5^{\prime}-\mathrm{H}\right), 1.50\left(\mathrm{~s}, 3 \mathrm{H}, \mathrm{CH}_{3}\right), 1.30\left(\mathrm{~s}, 3 \mathrm{H}, \mathrm{CH}_{3}\right)\end{array}$ \\
\hline $6 e$ & $\begin{array}{l}11.60(\mathrm{~s}, 1 \mathrm{H}, \mathrm{NH}), 11.46(\mathrm{~d}, J=2.04 \mathrm{~Hz}, 1 \mathrm{H}, \mathrm{NH}), 10.92(\mathrm{t}, J=5.40 \mathrm{~Hz}, 1 \mathrm{H}, \mathrm{NH}), 7.99(\mathrm{t}, J=1.89 \mathrm{~Hz}, 1 \mathrm{H}, \mathrm{ArH}), \\
7.88 \sim 7.52(\mathrm{~m}, 4 \mathrm{H}, \mathrm{ArH}+6-\mathrm{H}), 5.83\left(\mathrm{~d}, J=2.01 \mathrm{~Hz}, 1 \mathrm{H}, 1^{\prime}-\mathrm{H}\right), 5.66(\mathrm{dd}, J=2.13,7.95 \mathrm{~Hz}, 1 \mathrm{H}, 5-\mathrm{H}), 5.09(\mathrm{dd}, J= \\
\left.2.04,6.45 \mathrm{~Hz}, 1 \mathrm{H}, 2^{\prime}-\mathrm{H}\right), 4.86\left(\mathrm{dd}, J=4.32,6.33 \mathrm{~Hz}, 1 \mathrm{H}, 3^{\prime}-\mathrm{H}\right), 4.01 \sim 3.94\left(\mathrm{~m}, 2 \mathrm{H}, 5^{\prime}-\mathrm{H}\right), 1.50\left(\mathrm{~s}, 3 \mathrm{H}, \mathrm{CH}_{3}\right), 1.30(\mathrm{~s}, \\
\left.3 \mathrm{H}, \mathrm{CH}_{3}\right)\end{array}$ \\
\hline $6 f$ & $\begin{array}{l}11.55(\mathrm{~s}, 1 \mathrm{H}, \mathrm{NH}), 11.45(\mathrm{~d}, J=2.04 \mathrm{~Hz}, 1 \mathrm{H}, \mathrm{NH}), 10.94(\mathrm{t}, J=5.31 \mathrm{~Hz}, 1 \mathrm{H}, \mathrm{NH}), 7.94(\mathrm{dt}, J=2.49,4.74 \mathrm{~Hz}, 2 \mathrm{H}, \\
\mathrm{ArH}), 7.58(\mathrm{dt}, J=2.55,4.77 \mathrm{~Hz}, 2 \mathrm{H}, \mathrm{ArH}), 7.75(\mathrm{~d}, J=8.04 \mathrm{~Hz}, 1 \mathrm{H}, 6-\mathrm{H}), 5.82\left(\mathrm{~d}, J=2.04 \mathrm{~Hz}, 1 \mathrm{H}, 1^{\prime}-\mathrm{H}\right), 5.65(\mathrm{dd}, \\
J=2.16,5.76 \mathrm{~Hz}, 1 \mathrm{H}, 5-\mathrm{H}), 5.08\left(\mathrm{dd}, J=2.04,4.41 \mathrm{~Hz}, 1 \mathrm{H}, 2^{\prime}-\mathrm{H}\right), 4.84\left(\mathrm{dd}, J=4.41,6.96 \mathrm{~Hz}, 1 \mathrm{H}, 3^{\prime}-\mathrm{H}\right), 4.33 \sim 4.28 \\
\left(\mathrm{~m}, 1 \mathrm{H}, 4^{\prime}-\mathrm{H}\right), 4.02 \sim 3.89\left(\mathrm{~m}, 2 \mathrm{H}, 5^{\prime}-\mathrm{H}\right), 1.49\left(\mathrm{~s}, 3 \mathrm{H}, \mathrm{CH}_{3}\right), 1.29\left(\mathrm{~s}, 3 \mathrm{H}, \mathrm{CH}_{3}\right)\end{array}$ \\
\hline $6 \mathrm{~g}$ & $\begin{array}{l}12.09(\mathrm{~s}, 1 \mathrm{H}, \mathrm{NH}), 11.44(\mathrm{~s}, 1 \mathrm{H}, \mathrm{NH}), 10.59(\mathrm{t}, J=5.28 \mathrm{~Hz}, 1 \mathrm{H}, \mathrm{NH}), 7.74(\mathrm{~d}, J=8.04 \mathrm{~Hz}, 1 \mathrm{H}, 6-\mathrm{H}), 7.64 \sim 7.58(\mathrm{~m}, \\
1 \mathrm{H}, \operatorname{ArH}), 7.22(\mathrm{t}, J=8.22 \mathrm{~Hz}, 2 \mathrm{H}, \mathrm{ArH}), 5.82\left(\mathrm{~d}, J=1.89 \mathrm{~Hz}, 1 \mathrm{H}, 1^{\prime}-\mathrm{H}\right), 5.63(\mathrm{dd}, J=1.53,7.92 \mathrm{~Hz}, 1 \mathrm{H}, 5-\mathrm{H}), 5.09 \\
\left(\mathrm{dd}, J=1.86,6.39 \mathrm{~Hz}, 1 \mathrm{H}, 2^{\prime}-\mathrm{H}\right), 4.86\left(\mathrm{dd}, J=4.29,6.24 \mathrm{~Hz}, 1 \mathrm{H}, 3^{\prime}-\mathrm{H}\right), 4.34\left(\mathrm{t}, J=3.57 \mathrm{~Hz}, 1 \mathrm{H}, 4^{\prime}-\mathrm{H}\right), 4.05(\mathrm{~d}, J= \\
\left.5.25 \mathrm{~Hz}, 1 \mathrm{H}, 5^{\prime}-\mathrm{H}\right), 3.91\left(\mathrm{t}, J=2.07 \mathrm{~Hz}, 1 \mathrm{H}, 5^{\prime}-\mathrm{H}\right), 1.50\left(\mathrm{~s}, 3 \mathrm{H}, \mathrm{CH}_{3}\right), 1.30\left(\mathrm{~s}, 3 \mathrm{H}, \mathrm{CH}_{3}\right)\end{array}$ \\
\hline
\end{tabular}


续表

\begin{tabular}{|c|c|}
\hline Compd. & ${ }^{1} \mathrm{H}$ NMR $\left(\mathrm{DMSO}-d_{6}, 300 \mathrm{MHz}\right) \delta$ \\
\hline $6 \mathrm{~h}$ & $\begin{array}{l}11.82(\mathrm{~s}, 1 \mathrm{H}, \mathrm{NH}), 11.45(\mathrm{~d}, J=2.10 \mathrm{~Hz}, 1 \mathrm{H}, \mathrm{NH}), 10.72(\mathrm{t}, J=5.28 \mathrm{~Hz}, 1 \mathrm{H}, \mathrm{NH}), 7.75(\mathrm{~d}, J=8.07 \mathrm{~Hz}, 1 \mathrm{H}, 6-\mathrm{H}), \\
7.70 \sim 7.68(\mathrm{~m}, 1 \mathrm{H}, \mathrm{ArH}), 7.55 \sim 7.52(\mathrm{~m}, 1 \mathrm{H}, \mathrm{ArH}), 7.49 \sim 7.39(\mathrm{~m}, 2 \mathrm{H}, \mathrm{ArH}), 5.83\left(\mathrm{~d}, J=2.04 \mathrm{~Hz}, 1 \mathrm{H}, 1^{\prime}-\mathrm{H}\right), 5.64 \\
(\mathrm{dd}, J=2.19,7.98 \mathrm{~Hz}, 1 \mathrm{H}, 5-\mathrm{H}), 5.09\left(\mathrm{dd}, J=2.04,6.42 \mathrm{~Hz}, 1 \mathrm{H}, 2^{\prime}-\mathrm{H}\right), 4.85\left(\mathrm{dd}, J=4.35,6.39 \mathrm{~Hz}, 1 \mathrm{H}, 3^{\prime}-\mathrm{H}\right), 4.30 \sim \\
3.90\left(\mathrm{~m}, 2 \mathrm{H}, 5^{\prime}-\mathrm{H}\right), 1.50\left(\mathrm{~s}, 3 \mathrm{H}, \mathrm{CH}_{3}\right), 1.30\left(\mathrm{~s}, 3 \mathrm{H}, \mathrm{CH}_{3}\right)\end{array}$ \\
\hline $6 i$ & $\begin{array}{l}11.49(\mathrm{~s}, 1 \mathrm{H}, \mathrm{NH}), 11.40(\mathrm{~d}, J=1.98 \mathrm{~Hz}, 1 \mathrm{H}, \mathrm{NH}), 10.75(\mathrm{t}, J=5.55 \mathrm{~Hz}, 1 \mathrm{H}, \mathrm{NH}), 7.81(\mathrm{~d}, J=8.04 \mathrm{~Hz}, 1 \mathrm{H}, 6-\mathrm{H}), \\
7.70 \sim 7.59(\mathrm{~m}, 2 \mathrm{H}, \mathrm{ArH}), 7.39 \sim 7.29(\mathrm{~m}, 2 \mathrm{H}, \mathrm{ArH}), 5.82\left(\mathrm{~d}, J=2.01 \mathrm{~Hz}, 1 \mathrm{H}, 1^{\prime}-\mathrm{H}\right), 5.64(\mathrm{dd}, J=2.13,7.95 \mathrm{~Hz}, 1 \mathrm{H}, \\
5-\mathrm{H}), 5.09\left(\mathrm{dd}, J=1.95,6.42 \mathrm{~Hz}, 1 \mathrm{H}, 2^{\prime}-\mathrm{H}\right), 4.85\left(\mathrm{dd}, J=4.41,6.42 \mathrm{~Hz}, 1 \mathrm{H}, 3^{\prime}-\mathrm{H}\right), 4.35 \sim 4.29\left(\mathrm{~m}, 1 \mathrm{H}, 4^{\prime}-\mathrm{H}\right), 4.04 \sim \\
3.89\left(\mathrm{~m}, 2 \mathrm{H}, 5^{\prime}-\mathrm{H}\right), 1.50\left(\mathrm{~s}, 3 \mathrm{H}, \mathrm{CH}_{3}\right), 1.30\left(\mathrm{~s}, 3 \mathrm{H}, \mathrm{CH}_{3}\right)\end{array}$ \\
\hline $\mathbf{6 j}$ & $\begin{array}{l}11.83(\mathrm{~s}, 1 \mathrm{H}, \mathrm{NH}), 11.45(\mathrm{~d}, J=2.07 \mathrm{~Hz}, 1 \mathrm{H}, \mathrm{NH}), 10.86(\mathrm{t}, J=5.34 \mathrm{~Hz}, 1 \mathrm{H}, \mathrm{NH}), 8.32(\mathrm{dt}, J=2.10,4.83 \mathrm{~Hz}, 2 \mathrm{H}, \\
\mathrm{ArH}), 8.12(\mathrm{dt}, J=2.34,4.86 \mathrm{~Hz}, 2 \mathrm{H}, \mathrm{ArH}), 7.76(\mathrm{~d}, J=8.04 \mathrm{~Hz}, 1 \mathrm{H}, 6-\mathrm{H}), 5.82\left(\mathrm{~d}, J=2.07 \mathrm{~Hz}, 1 \mathrm{H}, 1^{\prime}-\mathrm{H}\right), 5.66(\mathrm{dd}, \\
J=2.19,5.79 \mathrm{~Hz}, 1 \mathrm{H}, 5-\mathrm{H}), 5.09\left(\mathrm{dd}, J=2.10,4.38 \mathrm{~Hz}, 1 \mathrm{H}, 2^{\prime}-\mathrm{H}\right), 4.86\left(\mathrm{dd}, J=4.41,6.01 \mathrm{~Hz}, 1 \mathrm{H}, 3^{\prime}-\mathrm{H}\right), 4.35 \sim 4.29 \\
\left(\mathrm{~m}, 1 \mathrm{H}, 4^{\prime}-\mathrm{H}\right), 4.04 \sim 3.92\left(\mathrm{~m}, 2 \mathrm{H}, 5^{\prime}-\mathrm{H}\right), 1.50\left(\mathrm{~s}, 3 \mathrm{H}, \mathrm{CH}_{3}\right), 1.30\left(\mathrm{~s}, 3 \mathrm{H}, \mathrm{CH}_{3}\right)\end{array}$ \\
\hline $6 k$ & $\begin{array}{l}11.45(\mathrm{~s}, 1 \mathrm{H}, \mathrm{NH}), 11.06(\mathrm{~s}, 1 \mathrm{H}, \mathrm{NH}), 10.86(\mathrm{t}, J=5.49 \mathrm{~Hz}, 1 \mathrm{H}, \mathrm{NH}), 7.89 \sim 7.61(\mathrm{~m}, 5 \mathrm{H}, \mathrm{ArH}+6-\mathrm{H}), 5.83(\mathrm{~d}, J= \\
\left.2.04 \mathrm{~Hz}, 1 \mathrm{H}, 1^{\prime}-\mathrm{H}\right), 5.65(\mathrm{dd}, J=1.26,7.95 \mathrm{~Hz}, 1 \mathrm{H}, 5-\mathrm{H}), 5.07\left(\mathrm{dd}, J=2.10,6.48 \mathrm{~Hz}, 1 \mathrm{H}, 2^{\prime}-\mathrm{H}\right), 4.86(\mathrm{dd}, J=4.38, \\
\left.5.82 \mathrm{~Hz}, 3^{\prime}-\mathrm{H}\right), 4.34 \sim 4.31\left(\mathrm{~m}, 1 \mathrm{H}, 4^{\prime}-\mathrm{H}\right), 4.06 \sim 3.89\left(\mathrm{~m}, 5 \mathrm{H}, 5^{\prime}-\mathrm{H}+\mathrm{Ar}-\mathrm{OCH}_{3}\right), 1.50\left(\mathrm{~s}, 3 \mathrm{H}, \mathrm{CH}_{3}\right), 1.30\left(\mathrm{~s}, 3 \mathrm{H}, \mathrm{CH}_{3}\right)\end{array}$ \\
\hline 61 & $\begin{array}{l}11.45(\mathrm{~s}, 1 \mathrm{H}, \mathrm{NH}), 11.21(\mathrm{~s}, 1 \mathrm{H}, \mathrm{NH}), 11.08(\mathrm{t}, J=5.25 \mathrm{~Hz}, 1 \mathrm{H}, \mathrm{NH}), 7.96 \sim 7.92(\mathrm{~m}, 2 \mathrm{H}, \mathrm{ArH}), 7.85 \sim 7.73(\mathrm{~m}, 1 \mathrm{H}, \\
6-\mathrm{H}), 7.04 \sim 6.94(\mathrm{~m}, 2 \mathrm{H}, \mathrm{ArH}), 5.83\left(\mathrm{~d}, J=2.13 \mathrm{~Hz}, 1 \mathrm{H}, 1^{\prime}-\mathrm{H}\right), 5.36(\mathrm{~d}, J=7.98 \mathrm{~Hz}, 1 \mathrm{H}, 5-\mathrm{H}), 5.08(\mathrm{dd}, J=2.13, \\
\left.6.48 \mathrm{~Hz}, 1 \mathrm{H}, 2^{\prime}-\mathrm{H}\right), 4.84\left(\mathrm{dd}, J=4.38,6.39 \mathrm{~Hz}, 1 \mathrm{H}, 3^{\prime}-\mathrm{H}\right), 4.36 \sim 4.27\left(\mathrm{~m}, 1 \mathrm{H}, 4^{\prime}-\mathrm{H}\right), 4.13 \sim 3.91\left(\mathrm{~m}, 4 \mathrm{H}, 5^{\prime}-\mathrm{H}+\right. \\
\left.\text { ArOCH}_{2} \mathrm{C}\right), 1.49\left(\mathrm{~s}, 3 \mathrm{H}, \mathrm{CH}_{3}\right), 1.37 \sim 1.03\left(\mathrm{~m}, 6 \mathrm{H}, \mathrm{CH}_{3}+\mathrm{ArOCCH}_{3}\right)\end{array}$ \\
\hline $6 \mathrm{~m}$ & $\begin{array}{l}11.54(\mathrm{~s}, 1 \mathrm{H}, \mathrm{NH}), 11.46(\mathrm{~s}, 1 \mathrm{H}, \mathrm{NH}), 10.89(\mathrm{t}, J=5.56 \mathrm{~Hz}, 1 \mathrm{H}, \mathrm{NH}), 7.75(\mathrm{~d}, J=8.04 \mathrm{~Hz}, 1 \mathrm{H}, 6-\mathrm{H}), 7.45 \sim 7.38(\mathrm{~m}, \\
2 \mathrm{H}, \mathrm{ArH}), 7.29 \sim 7.24(\mathrm{~m}, 2 \mathrm{H}, \mathrm{ArH}), 5.83\left(\mathrm{~d}, J=2.07 \mathrm{~Hz}, 1 \mathrm{H}, 1^{\prime}-\mathrm{H}\right), 5.63(\mathrm{~d}, J=7.98 \mathrm{~Hz}, 1 \mathrm{H}, 5-\mathrm{H}), 5.08(\mathrm{dd}, J=2.07, \\
\left.6.45 \mathrm{~Hz}, 1 \mathrm{H}, 2^{\prime}-\mathrm{H}\right), 4.85\left(\mathrm{dd}, J=4.44,6.42 \mathrm{~Hz}, 1 \mathrm{H}, 3^{\prime}-\mathrm{H}\right), 4.30\left(\mathrm{dd}, J=3.21,7.53 \mathrm{~Hz}, 1 \mathrm{H}, 4^{\prime}-\mathrm{H}\right), 4.01 \sim 3.90(\mathrm{~m}, 2 \mathrm{H}, \\
\left.5^{\prime}-\mathrm{H}\right), 2.36\left(\mathrm{~s}, 3 \mathrm{H}, \mathrm{CH}_{3}\right), 1.50\left(\mathrm{~s}, 3 \mathrm{H}, \mathrm{CH}_{3}\right), 1.30\left(\mathrm{~s}, 3 \mathrm{H}, \mathrm{CH}_{3}\right)\end{array}$ \\
\hline $6 n$ & $\begin{array}{l}11.44(\mathrm{~d}, J=2.07 \mathrm{~Hz}, 1 \mathrm{H}, \mathrm{NH}), 11.29(\mathrm{~s}, 1 \mathrm{H}, \mathrm{NH}), 11.05(\mathrm{t}, J=5.31 \mathrm{~Hz}, 1 \mathrm{H}, \mathrm{NH}), 7.91(\mathrm{~d}, J=1.80 \mathrm{~Hz}, 1 \mathrm{H}, \mathrm{ArH}), \\
7.88(\mathrm{t}, J=1.89 \mathrm{~Hz}, 1 \mathrm{H}, \mathrm{ArH}), 7.75(\mathrm{~d}, J=8.04 \mathrm{~Hz}, 1 \mathrm{H}, 6-\mathrm{H}), 7.54(\mathrm{~d}, J=1.83 \mathrm{~Hz}, 1 \mathrm{H}, \mathrm{ArH}), 7.52(\mathrm{~d}, J=1.86 \mathrm{~Hz}, \\
1 \mathrm{H}, \mathrm{ArH}), 5.82\left(\mathrm{~d}, J=2.10 \mathrm{~Hz}, 1 \mathrm{H}, 1^{\prime}-\mathrm{H}\right), 5.64(\mathrm{dd}, J=2.16,7.98 \mathrm{~Hz}, 1 \mathrm{H}, 5-\mathrm{H}), 5.80(\mathrm{dd}, J=2.13,6.48 \mathrm{~Hz}, 1 \mathrm{H}, \\
\left.2^{\prime}-\mathrm{H}\right), 4.84\left(\mathrm{dd}, J=4.35,6.39 \mathrm{~Hz}, 1 \mathrm{H}, 3^{\prime}-\mathrm{H}\right), 4.31\left(\mathrm{t}, J=2.70 \mathrm{~Hz}, 1 \mathrm{H}, 4^{\prime}-\mathrm{H}\right), 4.32 \sim 3.91\left(\mathrm{~m}, 2 \mathrm{H}, 5^{\prime}-\mathrm{H}\right), 1.49(\mathrm{~s}, 3 \mathrm{H}, \\
\left.\mathrm{CH}_{3}\right), 1.30\left(\mathrm{~d}, J=2.94 \mathrm{~Hz}, 12 \mathrm{H}, \mathrm{CH}_{3}+\operatorname{ArC}\left(\mathrm{CH}_{3}\right)_{3}\right.\end{array}$ \\
\hline 60 & $\begin{array}{l}11.49(\mathrm{~d}, J=5.55 \mathrm{~Hz}, 2 \mathrm{H}, \mathrm{NH}), 11.05(\mathrm{t}, J=5.43 \mathrm{~Hz}, 1 \mathrm{H}, \mathrm{NH}), 8.04(\mathrm{~d}, J=8.49 \mathrm{~Hz}, 2 \mathrm{H}, 6-\mathrm{H}+\mathrm{ArH}), 7.84 \sim 7.75(\mathrm{~m}, \\
5 \mathrm{H}, \mathrm{ArH}), 7.54 \sim 7.41(\mathrm{~m}, 3 \mathrm{H}, \mathrm{ArH}), 5.84\left(\mathrm{~d}, J=2.04 \mathrm{~Hz}, 1 \mathrm{H}, 1^{\prime}-\mathrm{H}\right), 5.66(\mathrm{~d}, J=8.01 \mathrm{~Hz}, 1 \mathrm{H}, 5-\mathrm{H}), 5.09(\mathrm{dd}, J=2.07, \\
\left.6.48 \mathrm{~Hz}, 1 \mathrm{H}, 2^{\prime}-\mathrm{H}\right), 4.86\left(\mathrm{dd}, J=4.38,6.24 \mathrm{~Hz}, 1 \mathrm{H}, 3^{\prime}-\mathrm{H}\right), 4.35 \sim 4.29\left(\mathrm{~m}, 1 \mathrm{H}, 4{ }^{\prime}-\mathrm{H}\right), 4.06 \sim 3.91\left(\mathrm{~m}, 2 \mathrm{H}, 5^{\prime}-\mathrm{H}\right), 1.50 \\
\left(\mathrm{~s}, 3 \mathrm{H}, \mathrm{CH}_{3}\right), 1.30\left(\mathrm{~s}, 3 \mathrm{H}, \mathrm{CH}_{3}\right)\end{array}$ \\
\hline
\end{tabular}

表 3 目标化合物的元素分析及红外光谱数据

Table 3 Elemental analysis and IR spectral data of target compounds

\begin{tabular}{|c|c|c|c|c|}
\hline \multirow{2}{*}{ Compd. } & \multicolumn{3}{|c|}{ Element analysis (cacld.) } & \multirow{2}{*}{$\mathrm{IR}(\mathrm{KBr}) v / \mathrm{cm}^{-1}$} \\
\hline & $\mathrm{C}$ & $\mathrm{H}$ & $\mathrm{N}$ & \\
\hline $6 a$ & $53.59(53.80)$ & $4.95(4.97)$ & $12.44(12.55)$ & $\begin{array}{l}3435,1690,1530,1469,2982,2943,1353,1267, \\
1210,1160,1052\end{array}$ \\
\hline $6 \mathbf{b}$ & $51.72(51.72)$ & $4.62(4.56)$ & $12.08(12.06)$ & $\begin{array}{l}3356,3210,3093,1692,1557,1521,1379,1284, \\
1210,1161,1094\end{array}$ \\
\hline $6 c$ & $51.72(51.62)$ & $4.62(4.60)$ & $12.08(12.05)$ & $\begin{array}{l}3344,1694,1555,1503,1458,2992,2941,1382, \\
1264,1239,1160,1088\end{array}$ \\
\hline 6d & $49.49(49.79)$ & $4.19(4.18)$ & $11.55(11.61)$ & $\begin{array}{l}3179,1694,1626,1579,1547,2989,2937,1384, \\
1277,1211,1182,1087\end{array}$ \\
\hline $6 e$ & $49.76(49.95)$ & $4.37(4.36)$ & $11.29(11.65)$ & $\begin{array}{l}3184,3049,1680,1569,1560,1528,2986,1377 \\
1249,1216,1174,1089\end{array}$ \\
\hline
\end{tabular}


续表

\begin{tabular}{|c|c|c|c|c|}
\hline \multirow{2}{*}{ Compd. } & \multicolumn{3}{|c|}{ Element analysis (cacld.) } & \multirow{2}{*}{$\operatorname{IR}(\mathrm{KBr}) v / \mathrm{cm}^{-1}$} \\
\hline & $\mathrm{C}$ & $\mathrm{H}$ & $\mathrm{N}$ & \\
\hline 6f & $49.87(49.95)$ & $4.67(4.40)$ & $11.45(11.65)$ & $\begin{array}{l}3431,1696,1551,1523,1457,2992,2939,1381, \\
1263,1214,1162,1090\end{array}$ \\
\hline $6 \mathrm{~g}$ & $46.44(46.61)$ & $3.98(3.91)$ & $10.85(10.87)$ & $\begin{array}{l}3164,1695,1588,1551,1515,2919,1379,1278, \\
1211,1174,1091\end{array}$ \\
\hline $6 \mathbf{h}$ & $45.64(45.72)$ & $4.02(4.03)$ & $10.63(10.66)$ & $\begin{array}{l}3211,3090,1690,1588,1531,2981,2940,1377, \\
1268,1214,1184,1158\end{array}$ \\
\hline $6 \mathbf{i}$ & $49.31(48.88)$ & $4.38(4.31)$ & $13.89(14.25)$ & $\begin{array}{l}3299,3220,1689,1575,1546,1524,2971,1373, \\
1277,1214,1150,1089\end{array}$ \\
\hline $6 \mathbf{J}$ & $48.77(48.88)$ & $4.46(4.31)$ & $14.53(14.25)$ & $\begin{array}{l}3317,1692,1529,1454,2992,2938,1377,1262, \\
1193,1159,1087\end{array}$ \\
\hline $6 \mathbf{k}$ & $52.67(52.65)$ & $5.05(5.08)$ & $11.67(11.76)$ & $\begin{array}{l}3318,3217,1694,1602,1515,1484,2987,1378, \\
1290,1226,1187,1160\end{array}$ \\
\hline 61 & $53.65(53.87)$ & $5.54(5.34)$ & $11.23(11.42)$ & $\begin{array}{l}3161,1694,1548,15003,1465,2984,2933,1381, \\
1324,1244,1181,10082\end{array}$ \\
\hline $6 \mathrm{~m}$ & $51.31(51.51)$ & $6.54(6.51)$ & $11.42(11.38)$ & $\begin{array}{l}3239,1681,1552,1521,2984,1377,1269,1216 \\
1181,1156\end{array}$ \\
\hline $6 n$ & $57.15(57.36)$ & $5.89(6.02)$ & $11.13(11.15)$ & $\begin{array}{l}3240,1694,1548,2964,1378,1268,1214,1161, \\
1089\end{array}$ \\
\hline 60 & $57.69(57.71)$ & $5.01(4.78)$ & $10.72(10.69)$ & $\begin{array}{l}3179,1694,1626,1579,1547,2989,2937,1383 \\
1277,1211,1181,1087\end{array}$ \\
\hline
\end{tabular}

生长后, 以十字交叉法测量各处理的菌落直径, 取其平 均值.

以校正后的空白对照和处理的菌落平均直径计算 抑制率，公式如下:

抑制率 $=\frac{\text { 对照菌落直径 } / \mathrm{mm} \text {-处理菌落直径 } / \mathrm{mm}}{\text { 对照菌落直径 } / \mathrm{mm}} \times 100 \%$ 所得化合物离体杀菌活性数据见表 4 .

\section{2 结果与讨论}

\section{1 合成方法}

\subsection{1 中间体的合成}

参照文献[12]方法合成中间体 $\mathbf{2}$ 时，作为此反应的 缚酸剂和溶剂, 吡啶用量非常大, 增加了反应后处理的 难度. 另外, 向吡啶溶液中加入对甲苯磺酰氯时, 反应 温度能快速上升至 $70{ }^{\circ} \mathrm{C}$, 易导致副产物 2',3'- $O$-异亚丙 基-5'-氯-5'-脱氧尿苷的产生, 从而影响反应收率. 因此, 在加入对甲苯磺酰氯时, 需用冰浴或者缓慢滴加等方式 以控制温度的迅速上升. 本文通过改善实验的反应体 系, 以二氯甲烷作为溶剂, 吡啶只作为缚酸剂, 配制成 混合溶剂, 回流反应. 在此体系中, 因为大量二氯甲烷 存在，使反应始终能够在不高于 $40{ }^{\circ} \mathrm{C}$ 左右进行，这样 就减少了副产物的生成. 另外，由于吡啶的用量减小,
表 4 目标化合物的离体杀菌活性结果 ${ }^{a}$

Table 4 Fungicidal activity in vitro of target compounds

\begin{tabular}{cccc}
\hline \multirow{2}{*}{ Compd. } & \multicolumn{3}{c}{ Inhibitory rate/\% } \\
\cline { 2 - 4 } & F. oxysporum & R. solani & P. bubak \\
\hline $\mathbf{6 a}$ & 44.0 & 17.0 & - \\
$\mathbf{6 b}$ & 48.3 & 53.9 & 53.1 \\
$\mathbf{6 c}$ & 30.7 & 40.2 & 34.5 \\
$\mathbf{6 d}$ & 57.1 & 53.2 & 47.6 \\
$\mathbf{6 e}$ & 33.2 & 39.4 & 62.0 \\
$\mathbf{6 f}$ & 50.5 & 46.6 & 40.5 \\
$\mathbf{6 g}$ & 48.3 & 48.7 & 62.2 \\
$\mathbf{6 h}$ & 36.6 & 18.9 & 51.7 \\
$\mathbf{6 i}$ & 36.4 & 38.4 & 79.5 \\
$\mathbf{6 J}$ & - & 29.1 & 65.5 \\
$\mathbf{6 k}$ & 42.8 & 58.4 & 72.4 \\
$\mathbf{6 1}$ & 54.5 & 83.9 & 81.1 \\
$\mathbf{6 m}$ & 63.8 & 40.5 & 97.2 \\
$\mathbf{6 n}$ & 53.9 & 39.4 & - \\
$\mathbf{6 0}$ & 66.7 & 73.00 & 41.7 \\
Polyoxin B & 75.8 & 87.3 & 10.0 \\
\hline${ }^{6}$ Mex
\end{tabular}

使后处理的难度降低，也提高了反应的收率. 反应体系 改进后，中间体 2 的收率在 $96 \%$ 以上.

\subsection{2 目标物的合成}

在目标物的合成中，中间体 $\mathbf{5}$ 的 $\mathrm{R}$ 基是反应能否顺 利进行的重要影响因素. 当 $\mathrm{R}$ 为烷基、叔丁基或烷氧基 
等给电子基团时，由于其共轭效应的影响，使异硫氰酸 酯的电子云密度增强, 使亲核加成反应更容易进行, 反 应在乙腈溶液中回流的时间在 $2.0 \sim 3.0 \mathrm{~h}$ 左右即进行完 毕. 当 $\mathrm{R}$ 为卤素钝化基团时, 反应比给电子取代基时困 难，一般回流 $6.0 \mathrm{~h}$ 方可进行完毕. 当 $\mathrm{R}$ 为硝基等吸电子 基团时, 由于其诱导效应的影响, 异硫氰酸酯的稳定性 降低. 若加热回流, 则易造成中间体 5 的分解使反应产 率降低, 此时温度需控制在 $55 \sim 60{ }^{\circ} \mathrm{C}$, 反应 $6.0 \sim 8.0 \mathrm{~h}$. 当 $\mathrm{R}$ 为相同取代基时，邻位取代的空间效应大于对位取 代, 对位取代反应产率略大于邻位取代.

\section{2 图谱分析}

目标化合物的 ${ }^{1} \mathrm{H}$ NMR 谱：化合物的活泼氢处于低 场; 尿苷环上的 5, 6 位氢原子受彼此影响, 大多规律地 裂分为 $\mathrm{dd}$ 峰及 $\mathrm{d}$ 峰, 化学位移分别在 $\delta 7.50$ 及 5.60 左 右; 苯环上的氢原子大多裂分为 $\mathrm{m}$ 峰, 其中对位取代化 合物多裂分为 $\mathrm{d}$ 峰,位移值分别在 $\delta 7.96 \sim 7.92$ 和 7.04 6.94 左右; 尿苷的呋喃糖环上的 $1^{\prime}, 2^{\prime}, 3^{\prime}, 4^{\prime}, 5^{\prime}$ 位氢的化 学位移值一般在 $\delta 5.80 \sim 3.90$ 之间.

目标化合物的 IR 谱: 化合物的 $\mathrm{N}-\mathrm{H}$ 伸缩振动吸收 峰位于 $3500 \sim 3200 \mathrm{~cm}^{-1}$; $\mathrm{C}=\mathrm{O}$ 伸缩振动位于 1780 及 $1690 \mathrm{~cm}^{-1}$ 附近; 1610,1520 及 $14800 \mathrm{~cm}^{-1}$ 附近的峰则对 应苯环的骨架振动. $\mathrm{CH}_{3}$ 的吸收峰在 2970 及 $1380 \mathrm{~cm}^{-1}$ 处; $1250 \mathrm{~cm}^{-1}$ 附近为 $\mathrm{C}-\mathrm{N}$ 的吸收峰, $1200 \mathrm{~cm}^{-1}$ 附近为 $\mathrm{C}=\mathrm{S}$ 伸缩振动峰.

\section{3 杀菌活性}

初步离体杀菌活性普篮结果表明, 目标化合物对棉 花枯萎病、水稻纹枯病和芦笋茎枯病表现出一定的抑制 效果. 大部分化合物对芦笋茎枯病表现出较高抑制活 性，抑制率在 50\%至 97\%之间，其中化合物 $6 \mathrm{~m}$ 和 61 的 抑制率分别为 $97.2 \%$ 和 $81.1 \%$, 化合物 $6 \mathbf{m}$ 与多氧霉素抑 制率相当. 对水稻纹枯病，化合物 61 的活性为 $83.9 \%$, 与多氧需素抑制率 $87.3 \%$ 相当. 但多数化合物的活性相 对较低, 原因可能有多方面包括立体效应、疏水性及电 性效应等因素影响, 具体原因还需要进一步研究探索. 从初步的构效关系分析发现: 苯环上的取代基类别及取 代位置对活性有一定的影响, 当 $\mathrm{R}$ 为卤素取代时, 活性 呈现出一定的规律, 为 $2,6-\mathrm{F}_{2}>\mathrm{Cl}>\mathrm{F}>\mathrm{Br}$; 当 $\mathrm{R}$ 相同时, 邻位取代时活性优于对位取代, 如对所有植物病原菌的 抑制效果, $6 \mathbf{b}>\mathbf{6 c}, \mathbf{6} \mathbf{i}>\mathbf{6} \mathbf{j}$; 当 $\mathrm{R}$ 为烷氧基和烷基等给电 子基团时, 对活性提升有利, 如 $6 \mathrm{k}, 6 \mathrm{l}, 6 \mathrm{~m}$ 及 60 . 但是, 当 $\mathrm{R}$ 为吸电子基团硝基时, 活性没有很大变化. 总体上
看，苯环上 $\mathrm{R}$ 基为给电子基时，活性优于吸电子基，这 对进一步的设计工作具有一定的指导意义.

本文所设计的目标化合物结构新颖, 并表现出较好 的杀菌活性，表明设计具有一定的合理性. 尤其是化合 物 61 和 $6 \mathrm{~m}$, 可作为先导化合物进行进一步优化研究.

\section{References}

[1] Gooday, G. W. In The Biochemistry of Cell Walls and Membranes in Fungi, Eds.: Goosey, M.; Kuhn, P.; Trinci, A. P., Springer-Verlag, Berlin, 1989, pp. $61 \sim 76$.

[2] Grosscurt, A. C.; Tipker, J. Pestic. Biochem. Physiol. 1980, 13, 249.

[3] (a) Isono, K.; Nagatsu, J.; Kawashiwa, Y.; Suzuki, S. Agric. Biol. Chem. 1965, 29, 848.

(b) Isono, K.; Asahi, K.; Suzuki, S. J. Am. Chem. Soc. 1969, 91, 7490 .

(c) Isono, K.; Suzuki, S. Heterocycles 1979, 13, 333.

[4] (a) Dähn, J.; Hagenmaier, H.; Höhner, H.; König, W. A.; Wolf, G.; Zähner, H. Arch. Microbiol. 1976, 107, 143.

(b) Hagenmaier, H.; Keckeisen, A.; Zähner, H.; König, W. A. Liebigs Ann. Chem. 1979, 10, 1494.

(c) König, W. A.; Hass, W.; Dehler, W.; Fiedler, H. P.; Zähner, H. Liebigs Ann. Chem. 1980, 4, 622.

[5] Khare, R. K.; Becker, J. M.; Naider, F. R. J. Med. Chem. 1988, 31, 650.

[6] Ephraim, C. Pest Manage. Sci. 2001, 57, 946.

[7] (a) Hori, M.; Misato, T. Agric. Biol. Chem. 1974, 38, 691. (b) Hori, M.; Misato, T. Agric. Biol. Chem. 1974, 38, 699.

(c) Wang, R.; Steensma, D. H.; Takaoka, Y.; Yun, J. W.; KaJimoto, T.; Wong, C. H. Bioorg. Med. Chem. 1997, 237, 476.

[8] Obi, K.; Uda, J.; Matsuda, A. Bioorg. Med. Chem. Lett. 2000, 10, 1451.

[9] Preeti, M. C.; Sunita, R. D. Bioorg. Med. Chem. 2009, 17, 2433.

[10] (a) Li, Y.; Li, B.-J.; Ling, Y.; Miao, H.-J.; Yang, X.-L. J. Agric. Food Chem. 2010, 58, 3037.

(b) Li, Y.; Li, B.-J.; Ling, Y.; Miao, H.-J.; Yang, X.-L. Chin. J. Org. Chem. 2011, 31, 1411 (in Chinese).

(李映, 李宝聚, 凌云, 苗宏健, 杨新玲, 有机化学, 2011, 31, 1411.)

[11] (a) Krishnamurthy, R. Pestic. Sci. 1998, 52, 145.

(b) Julio, J. C. J. Inorg. Biochem. 2005, 99, 1558.

(c) Zhou, W.-Q. Ph.D Dissertation, Nanjing University of Science and Technology, Nanjing, 2004 (in Chinese). (周为群, 博士论文, 南京理工大学, 南京, 2004.)

[12] Andrej, B.; Stanislav, G. Tetrahedron 2008, 64, 9093.

[13] Zhang, Z.-W. J. Central China Normal Univ. (Nat. Sci. ) 2007, 41, 88 (in Chinese).

(张正文, 华中师范大学学报 (自然科学版), 2007, 41, 88.)

[14] Chen, N.-C. Bioassay of Pesticides, Beijing Agricultural University Press, Beijing, 1991, p. 161 (in Chinese).

(陈年春, 农药生物测定技术, 北京农业大学出版社, 北京, 1991, p. 161.) 